\title{
Las metodologías de investigación en revisión: Análisis de redes sociales y producción textil local.
} Reaserch methodologies under revision: Social network analysis and textile production.

\author{
Mora del Pilar Castro \\ Universidad de Buenos Aires \\ moradelpilarcastro@gmail.com (ARGENTINA)
}

Recibido: 04.03.2015

Aceptado: 29.07.2015

\section{RESUMEN}

La reflexión sobre las metodologías sigue impulsando un amplio debate disciplinar que se actualiza de acuerdo a los nuevos desafíos que presenta el campo de la investigación. En esta ocasión, el presente artículo contribuye con algunas consideraciones teórico - metodológicas relativas al análisis antropológico de los sistemas de conocimiento local indígena y su transmisión vinculados con la producción de cultura material. En este marco, se presenta un caso comparativo entre dos comunidades en Argentina, abordado desde la perspectiva metodológica que brinda el Análisis de Redes Sociales.

En particular, se discuten aspectos de la investigación científica relativos al proceso que conforma la recolección de información, su procesamiento, la construcción del dato etnográfico y la elaboración del modelo de análisis. Así, se espera aportar a una discusión fundamental orientada a consolidar la aplicación de diferentes diseños metodológicos y sus usos en Antropología.

\section{PALABRAS CLAVE}

Metodología, dato etnográfico, producción textil, modelización, Análisis de Redes Sociales. 


\begin{abstract}
The reflection on research methodologies continues inspiring a wide disciplinary debate which updates according to new challenges in the research field. In this opportunity, this article contributes with some theoretical - methodological considerations related to the anthropological analysis of local and indigenous knowledge systems and its transmission regarding the production of material culture. Within this frame, we present a comparative case of study between two communities in Argentina from the Social Network Analysis perspective.

In particular, we argue on some aspects of scientific research including the course of data collection, its processing, the construction of ethnographic data, and the elaboration of an analytic model. Thus, we expect to contribute to a fundamental discussion pointing at consolidate the application of different methodological designs and its uses in Anthropology.
\end{abstract}

\title{
KEY WORDS
}

Methodology, ethnographic data, textile production, modeling, Social Network Analysis.

\section{INTRODUCCIÓN}

La discusión metodológica en Antropología, como en cualquier rama de la ciencia, posibilita la reflexión sobre aspectos específicos de la construcción del conocimiento de áreas particulares. A partir de la presentación de un caso de estudio, se propone ampliar el debate sobre la implementación de diseños de investigación novedosos que abordan problemas propios de la trayectoria disciplinar.

En este artículo se aborda el análisis del proceso de producción de textiles en contexto comunitario indígena y su relación con los sistemas de conocimiento local $\left(\mathrm{SLCI}^{1}\right)$ requeridos para realizar los pasos comprendidos en la cadena operativa de producción. El objetivo del artículo es discutir la implementación del Análisis de Redes Sociales (ARS) en el estudio de la transmisión de conocimientos para la producción, la organización comunitaria en relación al acceso de recursos y el análisis del diseño de las piezas de tejido. Se parte del supuesto que existen instancias específicas de transmisión de saberes al

${ }^{1}$ Se tomará la definición de este concepto a partir de la Declaratoria de la UNESCO al "cuerpo acumulativo y complejo de saberes, prácticas y representaciones que son mantenidas y desarrolladas por pueblos que habitan determinados lugares y que han interactuado con los mismos por generaciones, habiendo creado lazos de largo plazo y largo alcance con ese medio ambiente natural. Estos cuerpos de saberes constituyen "sistemas cognitivos" que funcionan dentro de interrelaciones más complejas que incluyen la cosmovisión, la espiritualidad y la lengua, entre otros elementos" (UNESCO 2002, Proyecto LINKS -Local and Indigenous Knowledge Systems). 
interior del grupo doméstico y fuera de él, así como se asumen patrones de diseño textil propio de cada región vinculados a la articulación variable entre los conocimientos y el acceso y procesamiento de los recursos disponibles.

La articulación teórica - metodológica para bordar esta problemática abarca tres perspectivas diferentes. En primer lugar, la Ecología Política (McGlade 1995) referida al estudio de la relación entre los grupos humanos y el ambiente en el que habitan. Luego, la línea de Paisaje Cultural (Hernández Llosas 2006) y Paisaje de Conocimiento (Strang 1997, 2008) que enfoca a la materialidad del espacio y a las representaciones que los diferentes grupos construyen en relación al mismo (Zedeño 2008). Finalmente, los SCLI (UNESCO 2002) cuya conceptualización apunta hacia los saberes, representaciones y prácticas mantenidas por los grupos sociales y su transmisión, considerando una escala temporal de largo alcance y el anclaje territorial que permite dicha transmisión.

El caso se plantea de forma comparativa a través del estudio en dos comunidades indígenas en Argentina, por un lado la Lofche Fvta Anekon (Comunidad Anekon Grande) localizada en la Región Sur de la provincia de Río Negro y, por el otro, la Comunidad Aborigen Negra Muerta, ubicada en el sector norte de la Quebrada de Humahuaca, provincia de Jujuy.

En la investigación realizada, se impulsa la articulación entre los métodos clásicos de la antropología con el Análisis de Redes Sociales (ARS), para sistematizar la información de trabajo etnográfico orientado a identificar los procesos de organización comunitarios en relación a la producción local textil. En este sentido, la recolección de información se basó en las sucesivas estadías en el trabajo de campo (conformado por ambas comunidades), las entrevistas a tejedoras/es e hilanderas/os y la observación participante de las diferentes actividades que componen la cadena operativa de la producción textil. Asimismo, se obtuvo información a partir del análisis de distintos tipos de fuentes secundarias, como censos de población, imágenes históricas y materiales de archivo. Luego de obtenida y organizada la información de base, se puso especial atención en la construcción del dato etnográfico, a partir de lo cual se procedió a la modelización de dichos datos.

El ARS ha tenido un impacto sumamente variable entre los científicos, por un lado ha sido entendido por algunos autores como un paradigma teórico y, por el otro, hay quienes lo reducen a un vocabulario técnico que da cuenta de patrones de vínculos y estructuras sociales. En este artículo se lo considera como una implementación específica del enfoque relacional para dar explicación a determinadas problemáticas de análisis social (Scott 2013). La construcción del modelo a partir del ARS permite no sólo sistematizar la información de campo sino también reorganizarla de manera tal que brinda una nueva aproximación a su lectura y al entrecruzamiento de datos con un enfoque relacional. Esta característica otorga un plus en dos sentidos: por un lado permite establecer puentes entre los dos casos analizados y por otro permite observar propiedades emergentes que surgen de la organización de la información.

Entonces, se plantea al análisis reticular como una herramienta teóricometodológica fundamental pues permite conceptualmente avanzar en la 
comprensión de las redes de la actividad a través de "mapear los actores en una red, tener la posibilidad de saber quiénes tienen una actuación central o periférica en un grupo, o quiénes ejercen mayor intermediación en una estructura" (Teves 2011).

Se presenta a continuación, en el acápite de Metodología, el proceso de relevamiento de información, la importancia de la construcción del dato etnográfico, la posibilidad de la modelización de los datos a partir del enfoque que brinda el ARS y se establecen las condiciones para la comparación de los casos propuestos. En Análisis se presentan las características de cada una de las redes construidas, su descripción técnica y el anclaje teórico para su análisis. En Discusión, se exponen algunos resultados de los dos tipos de comparación realizada entre las comunidades caso de estudio. Finalmente, las Conclusiones están planteadas los resultados obtenidos y las posibilidades a futuro en este campo de trabajo.

\section{METODOLOGÍA}

\subsection{Relevamiento de información y construcción del dato etnográfico}

En las investigaciones antropológicas, las fuentes de donde recolectar información suelen ser mayormente el trabajo de campo etnográfico y el apoyo con diferentes tipos de fuentes secundarias. Así, se comienza a generar un corpus creciente de materiales el cual es preciso ordenar de manera tal que se pueda distinguir la información relevante a los objetivos de estudio de aquella que es anecdótica.

Los criterios generales que permiten dicha distinción estarán alineados por el marco teórico construido a partir del cual se jerarquiza de manera diferencial los distintos aportes (Lozares Colina 2005). El proceso que hace que la información recolectada se convierta en un dato etnográfico recorre diversas decisiones metodológicas las cuales, articuladas, proporcionan la base del análisis.

En relación a la información obtenida del trabajo etnográfico para el caso presentado, se tuvo en cuenta la propuesta de Bernard (2006) en relación con que la presencia sostenida en el campo puede incidir en la colecta general de información, actuando de mecanismo de control de la fiabilidad de los datos obtenidos dado que "Presence builds trust. Trust lowers reactivity. Lower reactivity means higher validity of data" (Bernard 2006: 354).

La metodología de campo utilizada incorporó a la entrevista (en sus diferentes formas), la observación participante de diferentes situaciones que presente el contexto de análisis y la participación en la organización y apoyo a actividades con diferentes actores comunitarios. Todas estas instancias de interacción han permitido un acercamiento preferencial a los fenómenos de estudio y a la comprensión situada del análisis.

En este sentido, se acuerda con Teves en que la actividad textil va mucho más allá de la secuencia de operaciones que transforma las materias primas 
en una pieza textil. En esta instancia es relevante el estudio de los vínculos entre las personas que la realizan y las configuraciones establecidas en una red de vínculos. La información y las alternativas de interacción de la gente están afectadas por su lugar en la red de vínculos que se establecen mediante la actividad textil. La teoría de redes sociales y los procedimientos de esta metodología, describen importantes propiedades de la red, tales como centralización y cohesión entre los actores y ayudan al reconocimiento de patrones que pueden visualizarse en correspondencia con la estructura espacial (2011:6).

A partir de definir la población de interés, se fue delimitando la muestra a través de las posibilidades que se fueron dando en la situación de los trabajos etnográficos de conocer nuevas personas, visitar nuevos lugares y registrar nuevas piezas de tejido. Dentro de este tipo de muestreo dirigido (Sampieri et al. 1991), se utilizó uno de los tantos métodos de recolección de información, que se denomina Bola de Nieve a través del cual se comienza focalizando un actor o conjunto de actores, a cada uno de los cuales se les pregunta por algunos de sus lazos con otros actores (Hanneman 2000).

Este aspecto vinculado a las características de la muestra (tipo de muestreo, proporción en relación a la población total, instrumentos de medición, etc.) adquiere importancia cuando se discute sobre los criterios de confiabilidad, validez y representatividad de la misma en relación al alcance de las inferencias que se realizan a partir de ella por sobre el resto de la población considerada. Al respecto, Miceli (2011:23) plantea que hay dos aspectos a los cuales atender: la fiabilidad y la validez de los datos. Así, "la fiabilidad concierne al modo en que obtenemos los datos, y a los mecanismos más básicos por los cuales se puede asegurar la convergencia de resultados dadas las mismas condiciones de recolección. La validez está relacionada, en cambio, con cómo obtenemos conclusiones a partir de una masa de evidencias recolectada de manera fiable" (Ibíd.).

Las diferentes estrategias de recolección de información puestas en práctica a lo largo de 6 años de investigación estuvieron orientadas a indagar sobre los temas de interés. En relación a la transmisión de conocimiento, se preguntaba por quién le había enseñado a realizar la actividad (ya sea tejer, hilar, arreglar, recolectar materia prima, etc.). En relación al acceso a los recursos, se cuestionaba sobre cuáles eran los elementos utilizados y cuáles eran las otras unidades domésticas con las cuales se compartían (actualmente y en el pasado) los elementos necesarios para la elaboración de los tejidos. Finalmente, en lo vinculado al diseño de las piezas, se analizó cómo estaba compuesta la pieza a partir de sus agregados específicos de conocimiento ${ }^{2}$ (colores, figuras, formas) estableciendo la composición a partir de la co-presencia de los mismos.

${ }^{2}$ Esta denominación considera que la obtención de un color determinado, la elaboración de figuras decorativas en las piezas y la definición de la forma que lleva la misma tienen en su base procesos complejos que articulan conocimientos sobre los elementos y una manipulación concreta incluida en el proceso de producción. Por ello, se destaca en el concepto la noción de agregación. 
El tipo de información recolectada responde al enfoque relacional del análisis que luego es formalizada a través del ARS. En lugar de indagar por rasgos atributivos solamente, la aproximación elegida para analizar los aspectos de la producción textil local está basada no solamente en el análisis de las variables, sino especialmente en el análisis de las relaciones establecidas (Scott 2013: 5).

El trabajo de gabinete consistió en el procesamiento de la información de campo a través de fichas elaboradas especialmente para ordenar la selección y su consecuente volcado en una base de datos digital diseñada para este fin. Así, en estas fichas se concentró la información de las personas involucradas en las distintas etapas de la actividad textil, las características de las unidades domésticas que son y fueron el escenario de dicha actividad y, finalmente, los rasgos relevantes de las piezas textiles que conformaron la base material a analizar (Castro 2013a).

Toda la información mencionada sobre las personas, las características geográficas e históricas de los territorios comunitarios así como de las piezas textiles fue analizada con la herramienta del ARS utilizando una combinación de métodos particular para cada tipo de información (Bolívar, Martí y Lozares 2013).

\subsection{Modelización de los Fenómenos Sociales a Analizar}

La modelización de datos, como se ha discutido en otro lugar (Castro y Moreira 2008) es un puente fundamental y un modo particular de interacción entre la Teoría y el análisis de la realidad del caso de estudio. Consecuentemente, la construcción de un modelo es una manera dinámica de ordenamiento de información. La definición axiomática de "modelo" seguida aquí es la de "un esquema que permite configurar una serie de interacciones, entre sujetos u otras entidades formando así un universo finito de trabajo" (Reynoso 2006). Este universo finito de trabajo queda establecido a partir de los límites de cada una de las muestras para cada una de las redes, los cuales han sido pautados por el acceso en el trabajo etnográfico a las personas involucradas.

La construcción del modelo parte de identificar entidades relevantes para el análisis y relaciones existentes entre ellas (Butts 2008:14). Técnicamente, las entidades se denominan nodos y la relaciones entre ellos, lazos o vínculos. Los analistas de redes, "utilizan dos tipos de herramientas matemáticas para representar la información sobre los patrones de relaciones entre los nodos: grafos y matrices" (Hanneman 2000). Así, los datos se vuelcan en matrices, las cuales son conjuntos de elementos dispuestos en filas y columnas. Éstas se utilizan en análisis de redes para representar la adyacencia de cada nodo respecto de cada uno de los demás en la red. Una matriz de adyacencia es una matriz cuadrada, la cual se presenta con una forma de nodo por nodo $(\mathrm{i}=\mathrm{j})$, donde la presencia de vínculos se registran como elementos.

Al considerar las características del Análisis de Redes Sociales como una 
implementación específica del enfoque relacional, el tipo de dato relevante para armar un grafo es, justamente, aquel que brinde información para vincular (o no) a los nodos propuestos. De tal manera, los lazos proveen a las entidades oportunidades y al mismo tiempo restricciones potenciales sobre la dinámica planteada en un contexto determinado (Freeman 2004). En este mismo sentido, "el énfasis en la relación implica un marco teórico determinado que postule a los vínculos como eje central de sus hipótesis" (Díaz et al. 2010:4). Asimismo, de más está decir que los datos atributivos de cada nodo son también de mucha importancia para nutrir el análisis, pero el foco, una vez más, está puesto en las relaciones.

Una vez completas las matrices correspondientes, se traduce esa información a su representación gráfica a través de la utilización de software especializados ${ }^{3}$. Los grafos (compuestos por los nodos y los vínculos estipulados en las matrices) y las medidas resultantes de los análisis (medidas de los nodos, como centralidad de nodo, de intermediación y de cercanía así como las medidas de la red completa, por ejemplo la densidad, cantidad de componentes, la conectividad, la cohesión, configuración de subgrupos y sus variantes entre muchas otras) puntualizan la relacionalidad de la información y establecen la nueva base del análisis.

\subsection{Modelizar Permite Comparar}

Desde sus inicios, la Antropología se conformó tanto en sus perspectivas teóricas como en su metodología de análisis como una ciencia que apuntaba a la comparación entre diferentes grupos lo cual generó una larga lista de estudios que han permitido establecer parámetros comparativos entre diferentes sociedades a lo largo del globo. Comparaciones tanto diacrónicas en el sentido de poder comparar a un grupo en dos momentos diferentes del tiempo, comparaciones sincrónicas para poder comparar dos grupos diferentes en un mismo momento y, comparaciones que pretendían abarcar una integralidad de diferentes grupos que habilitaran apreciaciones de diferentes tipos (Adame 2006).

En el caso presentado aquí, la comparación de los procesos de producción textil local entre ambas comunidades se implementó a partir las características presentes en las tres redes conformadas para cada una de ellas en virtud de los aspectos significativos ya mencionados.

De manera complementaria e indispensable, se plantea la observación tanto de los cambios al interior de cada comunidad a través del tiempo como de las diferentes características que presentan los contextos socio-históricos de cada una de ellas. En este sentido, se entiende que la interpretación de los datos tiene sentido a partir de los contextos de acción y que la consideración de las

3 Por ejemplo, el UCINET (Borgatti et a. 2002), VISONE (2012), GEPHI (2008), ORA (2010). Para ver detalle de otras modelizaciones para redes personales, ver Maya y Holgado 2005. 
trayectorias históricas de ambas comunidades constituyen una escala de análisis más amplia y otorga una resignificación a los datos analizados para cada una de las redes, tanto para la investigación del fenómeno al interior del territorio comunitario como en su comparación.

En este marco, los puentes a través de los cuales se establece la comparación se elaboran a partir de la contrastación de las medidas resultantes de cada red, construida sobre cada tema para cada caso. En base a ellas se establecen puntos de contacto, lo cual permite visualizar: a) la estructura de los circuitos de enseñanza, b) los vínculos que presentan las unidades domésticas en su compartir elementos y c) la co-producción de los agregados específicos de conocimiento (ver infra).

Por otro lado, el ARS, como otros modelos provenientes de las teorías de la complejidad, permite observar las mencionadas propiedades emergentes que surgen de la organización de la información (Reynoso 2011). Éstas conforman nuevas dimensiones de análisis basadas en la no-linealidad de la lectura de los datos, posibilitando enriquecidas interpretaciones de los mismos, tales como: a) centralidad de ciertos nodos y su relevancia estructural en cada una de las redes, b) conformación de subgrupos de enseñanza - aprendizaje, c) co - ocurrencia de agregados específicos de conocimiento, d) caracterización de puntos de corte y de puentes para los circuitos de enseñanza y/ o co - ocurrencia de elementos, entre muchos otros.

\section{ANÁLISIS}

La aplicación del Análisis de Redes Sociales al estudio de la cultura material (textiles de elaboración comunitaria) y de los sistemas de conocimiento local indígena que hacen posible la realización de las piezas en territorio comunitario (a partir del conocimiento sobre la localización de los recursos en el territorio, significatividad de las combinaciones, circuitos de enseñanza, entre otros), se hace a partir de la generación de tres redes específicas para cada caso de estudio.

Se parte de la consideración que el armado de las redes propuestas contempla diferentes ejes de análisis cuya lectura conjunta permite aproximarnos de forma integral al proceso de producción local. La aplicación de ARS en este análisis brinda una herramienta para establecer cómo ciertas personas están interconectadas de manera tal que circula el conocimiento específico para producir piezas particulares de cultura material (Castro 2013b).

A continuación se describe cada una de las redes propuestas, sus rasgos estructurales y su articulación con el análisis a partir de los objetivos mencionados. Las imágenes son la expresión gráfica de cada una de las tres redes tanto para la Comunidad Anekon Grande como para la Comunidad Aborigen Negra Muerta ${ }^{4}$.

La primera red, está diseñada para mapear los vínculos entre las personas de la comunidad que hayan enseñado a otras a tejer y/o alguno de los pasos necesarios para la producción textil (red de enseñanza). Los nodos son las

4 El software utilizado para la graficación de la matriz es el VISONE (2012). 
personas y los vínculos establecen la relación de enseñanza y aprendizaje, tanto se haya dado al interior del grupo doméstico como por fuera de él. En términos técnicos, este grafo presenta vínculos orientados y simples (quién le enseñó a quién), presenta un solo componente (no hay nodos sin vinculaciones) y se diseñó con un solo tipo de nodos (red de un modo). El análisis de esta red está orientado a reconocer los circuitos de transmisión de los conocimientos locales sobre la actividad textil (incluidos en los $S C L I$ ). Adicionalmente, también indica las relaciones entre las distintas generaciones involucradas (formas de los nodos) así como identifica la relación entre los individuos presentes con su pertenencia a una unidad doméstica determinada (a través del color del nodo, a veces variado a raíz del cambio de unidad doméstica a través del tiempo).

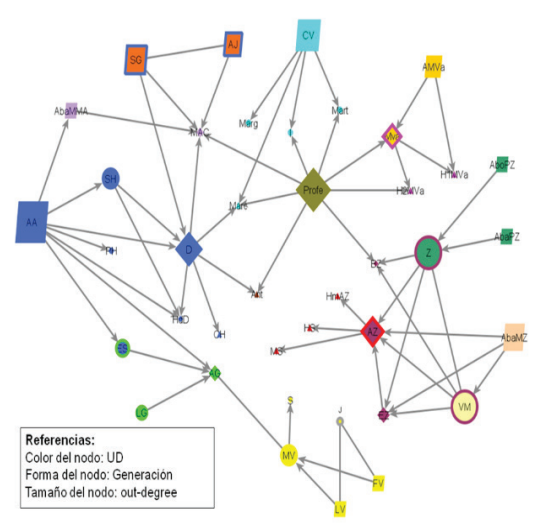

RED 1: Enseñanza (Negra Muerta). Generaciones y UD.

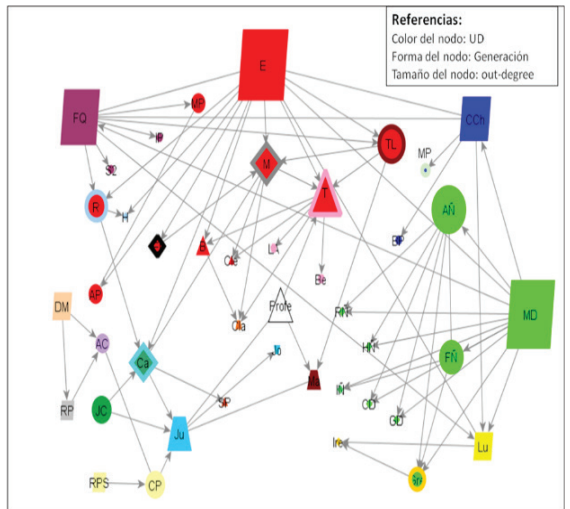

RED 1: Enseñanza (Anekon Grande). Generaciones y UD.

La segunda, establece a sus nodos como las unidades domésticas (presentes en el territorio comunitario y algunas fuera de él) y los lazos indican cuáles de ellas comparten elementos necesarios para realizar las piezas de tejido (red de elementos). En términos técnicos, este grafo se constituye como de un solo modo (dado que los nodos son de un solo tipo) y es unicomponente, por presentar todos los nodos unidos a través de algún tipo de vínculo. Asimismo, presenta vínculos binarios (dado que se entiende el compartir elementos como una actividad mutua) y multiplexados (a partir de considerar tres tipos de elementos para compartir: lana, colorantes, madera para el telar visualizando cada uno de ellos con lazo rojo, verdes y azul respectivamente). Finalmente, esta red admite el vínculo denominado loop (poco usual en el ARS), dado que se considera que las unidades domésticas se proveen a sí mismas, al menos, algunos de los elementos necesarios para realizar la actividad textil. El análisis de esta red está orientado a visualizar las relaciones entre las unidades domésticas a través de la producción local específica de piezas de tejido. A su vez, está diseñada para resaltar el aspecto espacial de la producción textil, es decir, reconocer el espacio 
territorial donde se lleva a cabo. Finalmente, el análisis de este grafo apunta también a indicar aspectos sobre el tipo de acceso a los recursos disponibles para la elaboración de las piezas en el marco que brinda la Ecología Política para entender la relación entre las sociedades y su territorio a lo largo del tiempo.

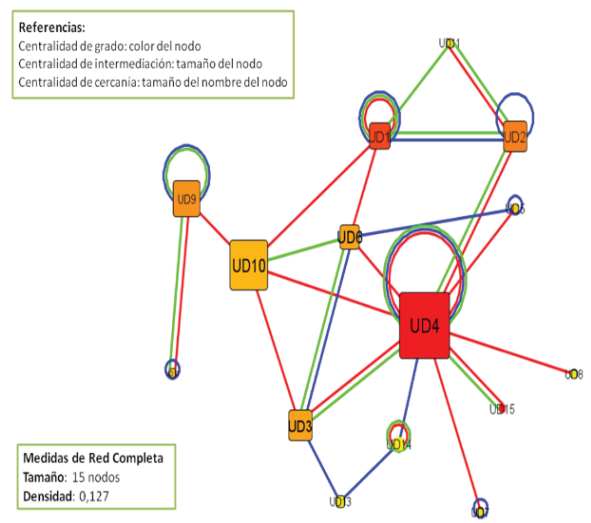

RED 2: Elementos (Negra Muerta). Centralidad.

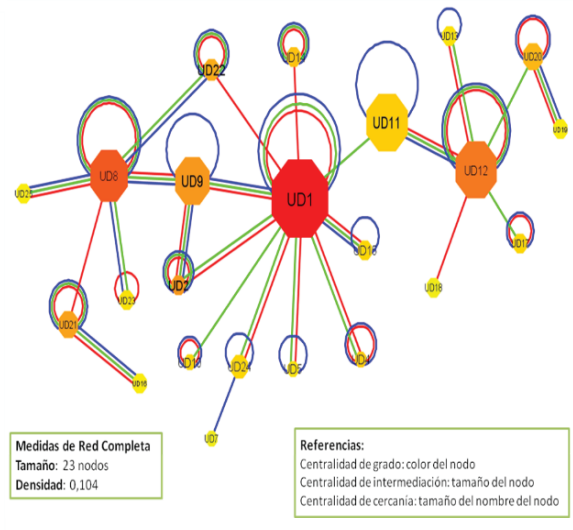

RED 2: Elementos (Anekon Grande). Centralidad.

La tercera red, se construye a partir de la co- ocurrencia de agregados específicos de conocimiento en las piezas textiles elaboradas en contexto comunitario (red de diseño). Los nodos se establecen a partir de la desagregación de los elementos que conforman la pieza de textil: su forma, sus colores y motivos (identificados a partir de la forma del nodo, circular, cuadrada, diamante respectivamente). Los lazos, se conforman a partir de la de la presencia conjunta de los elementos en una pieza particular. En términos técnicos, este grafo se constituye como de un solo modo (dado que los nodos son de un solo tipo) y es unicomponente, por presentar todos los nodos unidos a través de algún tipo de vínculo. Asimismo, presenta vínculos binarios (dado que se entiende la presencia conjunta en una misma pieza por definición no tiene una direccionalidad) y simples (dado que es un solo tipo de relación). El análisis de esta red está orientado a identificar los elementos de las prendas de la muestra en relación a la utilización de los recursos disponibles para su elaboración. Asimismo, se apunta a identificar el grado de frecuencia de aparición de los elementos (de los motivos, los colores y las formas) y repetición en la asociación entre ellos. La identificación de estos aspectos (qué elementos se utilizan, con qué frecuencia

5 Por ejemplo, si tomamos como parte de la muestra a una manta, que tiene forma rectangular, motivos de bandas y triángulos en colores natural y rojo. Los cinco nodos que se corresponden con estos elementos presentarán vínculos entre sí. 
aparecen y en qué medida se presenta una repetición en la co-ocurrencia de los mismos) hace permite evidenciar la importancia de ciertos diseños para las familias, su significación en la memoria social del grupo y su articulación con el Paisaje de Conocimiento constituido desde la producción textil.

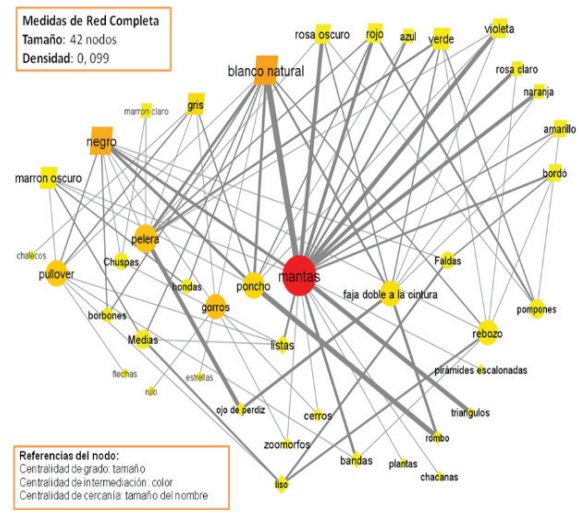

RED 3: Diseño (Negra Muerta). Centralidad.

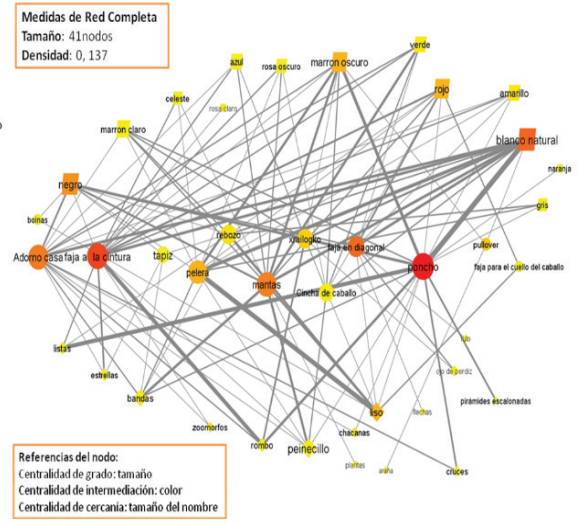

RED 3: Diseño (Anekon Grande). Centralidad.

En el Cuadro 1 se sintetizan las características técnicas para cada una de las redes propuestas. Asimismo, se apuntan los temas teóricos a los cuales se orienta el análisis de la lectura de las redes, tanto de manera separada como de manera conjunta.

\begin{tabular}{|c|c|c|c|c|}
\hline & Nodo & Lazo & $\begin{array}{l}\text { Caracteristica del } \\
\text { grafo }\end{array}$ & Temas abordados \\
\hline RED 1 & Personas & $\begin{array}{l}\text { A le enseña a } \\
\text { B }\end{array}$ & $\begin{array}{l}\text {-Orientado } \\
\text {-Simple } \\
\text {-Uni-componente } \\
\text {-Un modo }\end{array}$ & $\begin{array}{l}\text {-Relación entre las generaciones } \\
\text {-Identificación individuo- Unidad doméstica } \\
\text {-Circuitos de transmisión de los conocimientos-SCLI }\end{array}$ \\
\hline RED 2 & $\begin{array}{l}\text { Unidades } \\
\text { Domésticas }\end{array}$ & $\begin{array}{l}\text { A comparte } \\
\text { don B algún } \\
\text { elemento de } \\
\text { la producción } \\
\text { textil }\end{array}$ & $\begin{array}{l}\text {-Binario } \\
\text {-Multipexado } \\
\text {-Uni-componente } \\
\text {-Un modo }\end{array}$ & $\begin{array}{l}\text {-Vinculación entre las Unidades domésticas } \\
\text {-Espacialización de la Producción textil } \\
\text {-Acceso y organización de los recursos-Ecología Política }\end{array}$ \\
\hline RED 3 & $\begin{array}{l}\text { Agregados } \\
\text { especificos de } \\
\text { conocimientos }\end{array}$ & $\begin{array}{l}\text { A es } \\
\text { producidoen } \\
\text { conjunción } \\
\text { con B }\end{array}$ & $\begin{array}{l}\text {-Binario } \\
\text {-Simple } \\
\text { Uni-componente } \\
\text {-Un modo }\end{array}$ & $\begin{array}{l}\text {-Motivos de las prendas y elementos que las componen } \\
\text {-Repetición de la asociación } \\
\text {-Memoria social y significación-Paisajes de Conocimientos }\end{array}$ \\
\hline
\end{tabular}

Cuadro 1: síntesis de la caracterización de las redes propuestas 


\section{DISCUSIÓN}

Teniendo en cuenta la lectura conjunta de las tres redes para cada comunidad, se plantean las líneas a través de las cuales comparar ambos procesos de organización comunitaria orientados a la actividad textil así como las piezas de tejidos plasmados en las redes.

En este marco, se establece proponen dos tipos de comparación complementaria. Por un lado, se compara las redes 1 de cada comunidad entre sí, las redes 2 entre sí y lo mismo con las redes 3. Esta primera comparación apunta a contrastar la topografía de cada grafo particular. Por otro lado, se propone comparar las similitudes y diferencias a nivel de las tres redes para cada caso de estudio. De esta manera, se amplía la escala y se profundiza en las personas, las unidades domésticas y los agregados específicos de conocimiento, como así también en los lazos directos de enseñanza, de compartir los elementos y de coproducción de los rasgos de los diseños presentes (y pasados, si fuera el caso) en las comunidades. Finalmente se apunta a la identificación de distintas covariaciones distinguibles en la cultura material para cada caso.

En relación con la primera comparación mencionada, se puede afirmar que las topografías de la red de enseñanza para cada una de las comunidades presentan diferencias muy marcadas, vinculadas a la conformación de los circuitos de enseñanza de los pasos que componen la producción textil comunitaria. Estas diferencias radican, entre otras cosas, no solamente en la densidad o el tamaño de las redes sino en la importancia estructural que presentan ciertos nodos al momento de analizar la condición de intergeneracional de la transmisión del conocimiento. Las medidas de subgrupos marcan en ambas los espacios familiares de transmisión así como la identificación de personas ajenas a las comunidades que han tenido injerencia en el conocimiento actual de la actividad textil.

Asimismo, la comparación topográfica de la segunda red involucra el análisis de la localización espacial de las unidades domésticas y la dimensión temporal relativa al momento de ocupación de cada una de las unidades. En ambas puede observarse la centralidad de algunas unidades domésticas que tenían acceso a una mayor cantidad de recursos así como los compartían con otras. Asimismo, los integrantes de las familias que componen las comunidades tienen un fuerte lazo en la dinámica del compartir elementos para la producción textil ya sea que se ubiquen al interior del territorio comunitario como si éstas están localizadas por fuera del mismo, en pueblos o ciudades cercanas. También se ve en ambas comunidades el hecho de mantener vínculos con otros espacios territoriales a través de lazos de parentesco o compadrazgo que forman vías de acceso a recursos que no existen en la propia comunidad.

Por último, la comparación referida a la red tercera establece la amplia gama de combinaciones de estilos, formas y diseño de las piezas. A partir del análisis de las medidas de centralidad se identifican los motivos y los colores más utilizados y la frecuencia de aparición de las combinatorias particulares. Así, partiendo de este análisis y en conjunto con las medidas de subgrupos, 
se observan conjuntos de elementos que se reiteran, diferentes para cada comunidad, resultado de la combinatoria entre accesos a recursos específicos, conocimientos sobre su procesamiento y manufactura así como un tipo de uso de la prenda que denota distintos aspectos de la identidad local (Castro 2010).

La segunda comparación, vinculada al análisis de los casos integrando la lectura de las redes para cada comunidad en su conjunto, tiene una escala de análisis distinta a la precedente, dado que su base está conformada para observar desde una perspectiva más amplia la producción textil local y los sistemas de conocimiento que la hacen posible. Es decir, se analizan comparativamente a través de los resultados arrojados por el modelo de estudio de todas las redes a partir de los ejes de análisis.

De esta manera, se comparan las características de los circuitos de enseñanza a través de la observación de cuántas generaciones, quiénes son los nodos centrales de la muestra, qué nivel de transmisión entre las generaciones se presenta, qué rol han cumplido en dicha transmisión los talleres de enseñanza con una docente sobre tejido, que impacto ha tenido, qué diferencias presenta respecto de la enseñanza al interior de la unidad doméstica, qué rol juega la pertenencia a un mismo grupo familiar en relación a la transmisión y a los contenidos de la misma, etc.

Al mismo tiempo, se incorporan las características de la dinámica de compartir elementos que hacen posible la producción de esta específica muestra de cultura material, entre las cuales se encuentra el acceso diferencial a los recursos de acuerdo a la ubicación de la unidad doméstica y del espacio de uso familiar, los diferentes tipos de lazos entre esas mismas unidades domésticas que viabilizan ese acceso, el mantenimiento de esas relaciones entre unidades domésticas dentro y fuera del territorio comunitario, entre otros.

En lo referente a la co-producción de agregados específicos de conocimiento, se agrega a la comparación el análisis del producto terminado, con las características seleccionadas de las piezas como protagonistas, entre las cuales se puede mencionar la utilización de ciertos colores con ciertos tipos de prendas, la utilización de ciertos tipos de piezas en contextos cotidianos o ceremoniales específicos, la observación en la muestra del procesamiento concreto de los recursos disponibles y en qué medida la frecuencia de su utilización establece las marcas locales y qué nivel de significación conllevan ${ }^{6}$.

En síntesis, la aproximación relacional a los datos analizados a través de la implentación del ARS es una estrategia metodológica cuyos resultados son producto de las propiedades emergentes que se pueden desprender. Éstas conforman un espacio de análisis de diferente escala en relación a la información de base inicialmente recogida. Es decir, se recopiló, familia por familia los vínculos de enseñanza de las actividades involucradas en la actividad textil, esta información organizada de manera reticular hizo posible identificar los circuitos de transmisión de conocimiento presentes y pasados en las comunidades. De la misma manera, al identificar qué unidades domésticas compartían elementos

${ }^{6}$ Para más información, ver Castro (2013a) y Castro (2014). 
de la producción, emerge los canales de intercambio entre las personas y su relación con el territorio a partir de la disponibilidad y elección de los elementos para elaborar las piezas. Finalmente, de los vínculos entre los rasgos en cada pieza textil, se pueden establecer las mencionadas "marcas locales" propias de la elaboración comunitaria de las prendas.

\section{CONCLUSIÓN}

La construcción del conocimiento disciplinar se da a base de discusiones, respuestas, nuevas investigaciones, revisiones críticas, elaboraciones teóricas, implementaciones metodológicas y nuevas preguntas sobre las mismas o diferentes tópicos de interés.

¿Cómo se pasa de una oveja a un tapiz? ¿Cómo se obtiene tintura naranja a partir de unos frutos? ¿Por qué los cinturones de varón llevan guardas con triángulos en color natural? ¿Cómo se transmiten los saberes textiles? El análisis de los procesos de producción textil comunitaria y la transmisión del conocimiento local indígena son problemáticas amplias con muchas aristas que atender. El análisis de las mismas en los casos de estudio propuestos han permitido avanzar en la identificación de algunos rasgos vinculando la relación entre esas comunidades a lo largo del tiempo con los espacios territoriales habitados.

Así, se ha podido identificar los circuitos de transmisión de los conocimientos involucrados en la actividad textil y el papel que dicha transmisión juega en la cohesión social, la centralidad de ciertas personas y la emergencia de claros subgrupos que re-organizan en análisis de las relaciones sociales al interior de las comunidades.

Por otro lado, el análisis del producto final de todo el proceso de producción -la pieza textil- brinda un espacio de análisis muy provechoso en el cual se han podido establecer la utilización de ciertos elementos, la asociación entre ellos y sus frecuencias. La elaboración de las redes y el análisis de sus métricas ha permitido identificar la emergencia de las "marcas locales" en los diseños y la importancia que su realización conlleva.

La articulación metodológica que implica la implementación del ARS como modelo de análisis ha conformado el centro de la discusión a través de la descripción de sus características principales, las decisiones tomadas en la recolección de la información y la construcción de los datos etnográficos para arribar a la modelización del problema de estudio. En particular, la aplicación del ARS al análisis de la producción textil comunitaria y a la transmisión del conocimiento local, ha contribuido, en principio, a establecer una ampliación del campo de aplicabilidad del ARS a temas que antes no habían sido abordados con este enfoque. Asimismo, se han conformando nuevos pisos de análisis a partir de las propiedades emergentes resultado de proceso de investigación presentado aquí.

Se ha discutido la comparación entre las redes a partir de la topografía de los 
grafos, estableciendo los rasgos característicos de cada caso: las métricas de los nodos y las de red completa apuntaron identificar particularidades estructurales de la actividad textil. Conjuntamente, se ha planteado la comparación entre los casos a partir de los resultados analizados de manera integral para cada uno de ellos.

El hecho que la construcción de un modelo analítico con las características del ARS permita establecer parámetros claros y replicables de comparación de casos es una propiedad muy importante, la cual brinda, por un lado, respuestas de investigación propias de este diseño particular, y por otro, amplía el debate metodológico sobre los criterios de validez y confiabilidad, fundamentales para la elaboración de modelos sólidos.

Se afirma con Teves et al. (2011) que "el potencial articulador del ARS permite utilizar metodologías clásicas, sistematizar más datos y ver dinámicas". Es decir, la potencialidad que esta herramienta posee para la sistematización e interpretación de los datos brinda una oportunidad para reformular la información en el mismo proceso de la elaboración de las redes. Los avances en este sentido aportan a la consolidación de las problemáticas como objeto de interés. Se espera haber contribuido a esta construcción a partir del caso presentado con la intención de continuar el debate.

\section{AGRADECIMIENTOS}

Quisiera agradecer a mis directoras, la Dra. María Isabel Hernández Llosas y la Dra. Diana Lenton por haberme guiado a lo largo de los años de investigación. Agradezco, a su vez, a mis compañeros y compañeras de los equipos en los que participo por su ayuda y valiosas sugerencias. Asimismo, quisiera agradecer a quienes han evaluado este artículo para su publicación, por sus agudas recomendaciones y sugerencias. Por supuesto que cualquier falencia que recaiga sobre el trabajo realizado es de mi exclusiva responsabilidad.

\section{BIBLIOGRAFÍA}

ADAME, M. Á. (2006): "En torno a nuevas corrientes, nuevas temáticas y nuevos sujetos de estudio de la antropología sociocultural". Cuicuilco, vol. 13, núm. 37, mayo-agosto, 2006, pp. 25-57, Escuela Nacional de Antropología e Historia. México.

BERNARD, R. (2006): Research methods in anthropology: qualitative and quantitative approaches. Oxford: Altamira Press.

BOLIVAR, M., MARTÍ, J. y LOZARES, C (2013) “Aplicaciones de los métodos mixtos al análisis de las redes personales de la población inmigrada”. En EMPIRIA. Revista de Metodología de Ciencias Sociales. N.o 26, julio-diciembre, 2013, pp. 89-116. ISSN: 1139-5737, DOI: 10.5944/empiria.26.7154

BORGATTI, S., EVERETT, M. y L.FREEMAN (2002): UCINET for Windows: Software for Social Network Analysis. Harvard: Analytic Technologies. 
BUTTS, C. (2008): "Social network analysis: A methodological introduction". Asian Journal of Social Psychology, vol. 11, 13-41.

CASTRO, M. (2014 en prensa): "Transmisión de Conocimiento y Producción Textil: memoria social desde una perspectiva del análisis de redes sociales a multinivel". En Revista Redes. Disponible en http://revista-redes.rediris.es/ webredes/

$\left(2013^{\mathrm{a}}\right)$ : Sistemas de Conocimiento local indígena y Cultura Material. Una investigación (antropo-arqueológica) comparativa en dos comunidades indígenas de Noroeste y Patagonia. Tesis de Doctorado en Antropología, Universidad de Buenos Aires. Argentina.

(2013b): "Paisaje y Producción textil: un abordaje desde la Ecología Política". Anuario del Departamento de Arqueología, volumen especial. Escuela de Antropología, Facultad de Humanidades y Artes, Universidad Nacional de Rosario. Argentina.

(2010): “Conformación de Identidades Locales y Cultura Material: un Análisis desde los 'Sistemas de Conocimiento Local Indígena' y la Producción de Arte Textil”. Espaço Ameríndio, Porto Alegre, v. 4, n. 2, p. 206-232. (Disponible on line. http://seer.ufrgs. br/EspacoAmerindio/index bajado el 01.06.2013).

CASTRO, M. y MOREIRA, M. (2008): La modelización como una vía para lidiar con la realidad en la investigación científica. Trabajo presentado en el IX Congreso Argentino de Antropología Social "Fronteras de la Antropología". Realizado en Posadas, Misiones. Argentina.

DÍAZ, D., MACTAS, M., SCANIO, P., PICCARDI, G., QUINTEROS, R., DOMÍNGUEZ, M., MOREIRA, M. (2010): “Algunos presupuestos metodológicos del Análisis de Redes Sociales”. (Disponible en www.antropocaos. com.ar bajado el 01 de mayo de 2013)

FREEMAN, L. (2004): The Development of Social Network Analysis: A Study in the Sociology of Science. Vancouver: Empirical Press.

HANNEMAN, R. (2000): Introducción a los métodos del análisis de redes sociales. (http://revistaredes.rediris.es/webredes/textos)

HERNÁNDEZ LLOSAS, M. I. (2006): "Diversidad cultural, patrimonio e identidad en Argentina". En La dimensión social del patrimonio. Centro Internacional para la Conservación del Patrimonio, Buenos Aires.

Karlsruhe Institute of Technology (2012): VISONE (visual social networks). (Disponible en http://visone.info/)

LOZARES COLINA, C. (2005) "Bases sociometodológicas para el Análisis de Redes Sociales, ARS”. EMPIRIA. Revista de Metodología de Ciencias Sociales. N.»10, julio-diciembre, 2005, pp. 09-36. ISSN: 1139-5737

MAYA, I. y HOLGADO, D (2005) "Lazos fuertes y proveedores múltiples de apoyo: comparación de dos formas de representación gráfica de las redes personales". EMPIRIA. Revista de Metodología de Ciencias Sociales. N.»10, julio-diciembre, 2005, pp. 107-127. ISSN: 1139-5737

MCGLADE, J. (1995): "Archaeology and the Ecodynamics of Human-Modified landscapes". En Antiquity, 69, 113-132.

MICELI, J. (2011): "Formas de Control Cruzado de datos en la investigación: algunos avances sistematizadores”. En X Congreso Argentino de Antropología Social, Buenos Aires.

REYNOSO, C. (2006): Complejidad y Caos: una exploración antropológica. Editorial $\mathrm{SB}$, Buenos Aires. 
(2011): Redes Sociales y Complejidad. Modelos interdisciplinarios en la gestión sostenible de la sociedad y la cultura. Buenos Aires. Editorial SB.

SAMPIERI, Roberto, FERNÁNDEZ COLLADO, Carlos y Pilar BAPTISTA LUCIO (1991): Metodología de la Investigación. México. McGraw-Hill.

SCOTT,J. (2013): Social Network Analysis. SAGE. Londres.

STRANG, V. (1997): Uncommon Ground: Cultural Landscapes and Environmental Values. Oxford. Berg.

(2008): Uncommon ground: Landscape as Social Geography". En Handbook of Landscape Archaeology, pp. 51-59. David, B. and Thomas, J. (eds.) Left Coast Press, California.

TEVES, L. (2011): El Estudio Etnográfico de la Actividad Textil como aporte a la Caracterización del Modo de Vida en el Pueblo de Molinos y zona de influencia (Provincia de Salta). Tesis de Doctorado en Antropología, UNLP. Argentina.

TEVES, L., CRIVOS, M. y A. LAMARCHE (2011): “Cambios teórico-metodológico en una investigación etnográfica. El potencial articulador del ARS”. En Actas del X Congreso Argentino de Antropología Social, Buenos Aires.

UNESCO (2002): Paris: Local and Indigenous Knowledge Systems (LINKS). Disponible en www.unesco.org/links

ZEDEÑO, M. (2008): “The Archaeology of Territory and Territoriality". En Handbook of Landscape Archaeology, pp. 210-217. David, B. and Thomas, J. (eds.) Left Coast Press, California. 
